\title{
Static two-player evasion-interrogation games with uncertainty*
}

\author{
H.T. BANKS, S.L. GROVE, K. ITO and J.A. TOIVANEN \\ Center for Research in Scientific Computation, North Carolina State University \\ Raleigh, NC 27695-8205
}

E-mails: htbanks@ncsu.edu / slgrove@ncsu.edu / kito@math.ncsu.edu / jatoivan@ncsu.edu

\begin{abstract}
We consider electromagnetic evasion-interrogation games wherein the evader can use ferroelectric material coatings to attempt to avoid detection while the interrogator can manipulate the interrogating frequencies (wave numbers) and angles of incidence of the interrogating inputs to enhance detection and identification. The resulting problems are formulated as two player games in which one player wishes to minimize the reflected signal while the other wishes to maximize it. Simple deterministic strategies are easily defeated and hence the players must introduce uncertainty to disguise their intentions and confuse their opponent. Mathematically, the resulting game is carried out over spaces of probability measures which in many cases are appropriately metrized using the Prohorov metric.
\end{abstract}

Mathematical subject classification: 49N75, 91A05, 91A10, 91A60, 78A30, 78A46.

Key words: pursuit-evasion games with uncertainty, electromagnetic interrogation, inverse scattering.

\section{Introduction}

In [8] the authors demonstrated that it is possible to design ferroelectric materials with appropriate dielectric permittivity and magnetic permeability to significantly attenuate reflections of electromagnetic interrogation signals from highly

\#688/06. Received: 01/IX/06. Accepted: 04/X/06.

* On the Occasion of the $25^{\text {th }}$ Anniversary of the Laboratório Nacional de Computação Científica (LNCC), March 21-24, 2006, Rio de Janeiro, Brazil. 
conductive targets such as airfoils and missiles. This was done under assumptions that the interrogating input signal is uniformly likely to come from a sector of interrogating angles $\alpha \in\left[\alpha_{0}, \alpha_{1}\right]\left(\alpha=\frac{\pi}{2}-\phi\right.$ where $\phi$ is the angle of incidence of the input signal) but that the evader has knowledge of the interrogator's input frequency or frequencies (denoted in the presentations below as the interrogator design frequencies $I_{D}$ ). These results were further sharpened and illustrated in [9] where a series of different material designs were considered to minimize over a given set of input design frequencies $I_{D}$ the maximum reflected field from input signals. In addition, a second critical finding was obtained in that it was shown that if the evader employed a simple counter interrogation design based on a fixed set (assumed known) of interrogating frequencies $I_{D}$, then by a rather simple counter-counter interrogation strategy (use an interrogating frequency little more than $10 \%$ different from the assumed design frequencies), the interrogator can easily defeat the evader's material coatings counter interrogation strategy to obtain strong reflected signals.

From the combined results of $[8,9]$ it is thus rather easily concluded that the evader and the interrogator must each try to confuse the other by introducing significant uncertainty in their design and interrogating strategies, respectively. This concept, which we refer to as mixed strategies in recognition of previous contributions to the literature on games (von Neumann's finite mixed strategies to be explained below), leads to two player non-cooperative games with probabilistic strategy formulations. These can be mathematically formulated as two sided optimization problems over spaces of probability measures, i.e., minmax games over sets of probability measures. The purpose of this paper is to provide a mathematically precise formulation of such a class of two sided optimization problems and to discuss our initial computational efforts on such problems. Approximation methods are introduced and their computational efficacy is demonstrated with several simple examples.

\section{Problem formulation}

We consider electromagnetic interrogation of objects in the context of minmax evader-interrogator games where each player has uncertain information about the adversary's capabilities. The minmax cost functional is based on reflected 
fields from an object such as an airfoil or missile and can be computed in one of several ways $[8,9]$.

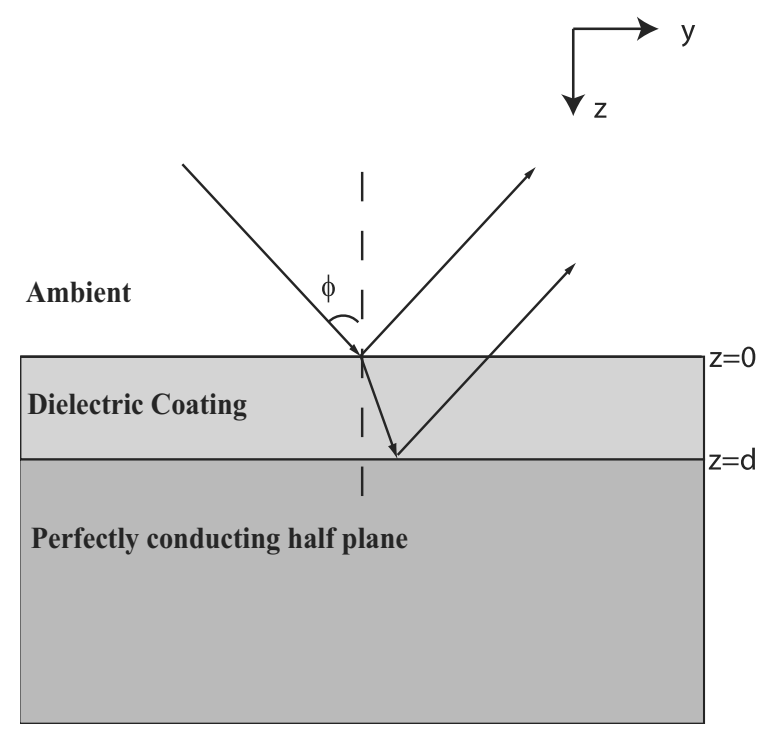

Figure 1 - Interrogating high frequency wave impinging (angle of incidence $\phi$ ) on coated (thickness $d$ ) perfectly conducting surface.

The simplest is the reflection coefficient based on a simple planar geometry (e.g., see Figure 1) using Fresnel's formula for a perfectly conducting half plane which has a coating layer of thickness $d$ with dielectric permittivity $\epsilon$ and magnetic permeability $\mu$. A normally incident $(\phi=0)$ electromagnetic wave with the frequency $f$ is assumed to impinge the half plane. Then the corresponding wavelength $\lambda$ in air is $\lambda=c / f$, where the speed of light is $c=0.3 \times 10^{9}$. The reflection coefficient $R$ for the wave is given by

$$
R=\frac{a+b}{1+a b}
$$

where

$$
a=\frac{\epsilon-\sqrt{\epsilon \mu}}{\epsilon+\sqrt{\epsilon \mu}} \quad \text { and } \quad b=e^{4 i \pi \sqrt{\epsilon \mu} f d / c} .
$$

This expression can be derived directly from Maxwell's equation by considering the ratio of reflected to incident wave for example in the case of parallel polarized $\left(T E_{x}\right)$ incident wave (see $\left.[8,20]\right)$. 
An alternative and much more computationally intensive approach (which may be necessitated by some target geometries) employs the far field pattern for reflected waves computed directly using Maxwell's equations. In two dimensions, for a reflecting body $\Omega$ with coating layer $\Omega_{1}$ and computational domain $\Pi$ with an interrogating plane wave $E^{(i)}$, the scattered field $E^{(s)}$ satisfies the Helmholtz equation [15]

$$
\begin{array}{ll}
\nabla \cdot\left(\frac{1}{\mu} \nabla E^{(s)}\right)+\epsilon \omega^{2} E^{(s)}=-\nabla \cdot\left(\frac{1}{\mu} \nabla E^{(i)}\right)-\epsilon \omega^{2} E^{(i)} & \text { in } \Pi \backslash \bar{\Omega} \\
E^{(s)}=-E^{(i)} & \text { on } \partial \Omega \\
{\left[\frac{1}{\mu} \frac{\partial E}{\partial n}\right]=[E]=0} & \text { on } \partial \Omega_{1} \backslash \partial \Omega \\
\frac{\partial E^{(s)}}{\partial n}-i k E^{(s)}-\frac{i}{2 k} \frac{\partial^{2} E^{(s)}}{\partial s^{2}}=0 & \text { on } \partial \Pi \\
\frac{\partial E^{(s)}}{\partial s}-i k \frac{3}{2} E^{(s)}=0 & \text { at } C,
\end{array}
$$

where the Silver-Müller radiation condition has been approximated by a secondorder absorbing boundary condition on $\partial \Pi$ as described in $[2,17,18]$. The vectors $n$ and $s$ denote the normal and tangential directions on the boundary $\partial \Pi$, respectively, and $C$ is the set of the corner points of $\Pi$. Here [ $\cdot]$ denotes the jumps at interfaces. The incident field in air is given by

$$
E^{(i)}=e^{i k\left(x_{1} \cos \alpha+x_{2} \sin \alpha\right)},
$$

where $\alpha$ is the interrogation angle $\left(\alpha=\frac{\pi}{2}-\phi\right)$ and $k=2 \pi f / c=\omega / c$ is the interrogation wave number corresponding to the interrogating frequency $f$. The corresponding far field pattern is given by $[8,15]$

$$
\begin{gathered}
F_{\alpha}(\alpha+\pi ; \epsilon, \mu, \alpha, f) \\
=\lim _{r \rightarrow \infty}\left(\sqrt{8 \pi k r} e^{-i(k r+\pi / 4)} E^{(s)}(r \cos (\alpha+\pi), r \sin (\alpha+\pi) ; \epsilon, \mu, \alpha, f)\right),
\end{gathered}
$$

where $E^{(s)}\left(x_{1}, x_{2} ; \epsilon, \mu, \alpha, f\right)$ is the scattered electromagnetic field. This can be used as a measure of the reflected field intensity instead of the reflection coefficient $R$ of (1)-(2).

The evader and the interrogator are each subject to uncertainties as to the actions of the other. The evader wants to choose a best coating design (i.e., best 
$\epsilon$ 's and $\mu$ 's ) while the interrogator wants to choose best angles of interrogation $\alpha$ and interrogating frequencies $f$. Each player must act in the presence of incomplete information about the other's action. Partial information regarding capabilities and tendencies of the adversary can be embodied in probability distributions for the choices to be made. That is, we may formalize this by assuming the evader may choose (with an as yet to be determined set of probabilities) dielectric permittivity and magnetic permeability parameters $(\epsilon, \mu)$ from admissible sets $\mathcal{E} \times \mathcal{M}$ while the interrogator chooses angles of interrogation and interrogating frequencies $(\alpha, f)$ from sets $\mathcal{A} \times \mathcal{F}$. The formulation here is based on the mixed strategies proposals of von Neumann [1,22, 23] and the ideas can be summarized as follows. The evader does not choose a single coating, but rather has a set of possibilities available for choice. He only chooses the probabilities with which he will employ the materials on a target. This, in effect, disguises his intentions from his adversary. By choosing his coatings randomly (according to a best strategy to be determined in, for example, a minmax game), he prevents adversaries from discovering which coating he will use-indeed, even he does not know which coating will be chosen for a given target. The interrogator, in a similar approach, determines best probabilities for choices of frequency and angle in the interrogating signals. Note that such a formulation tacitly assumes that the adversarial relationship persists with multiple attempts at evasion and detection.

The associated minmax problem consists of the evader choosing distributions $P_{e}(\epsilon, \mu)$ over $\mathcal{E} \times \mathcal{M}$ to minimize the reflected field while the interrogator chooses distributions $P_{i}(\alpha, f)$ over $\mathcal{A} \times \mathcal{F}$ to maximize the reflected field. If $B_{R}(\epsilon, \mu, \alpha, f)$ is the chosen measure of reflected field and $\mathcal{P}_{e}=\mathcal{P}(\mathcal{E} \times \mathcal{M})$ and $\mathcal{P}_{i}=\mathcal{P}(\mathcal{A} \times \mathcal{F})$ are the corresponding sets of probability distributions or measures over $\mathcal{E} \times \mathcal{M}$ and $\mathcal{A} \times \mathcal{F}$, respectively, then the cost functional for the minmax problem can be defined by

$$
J\left(P_{e}, P_{i}\right)=\int_{\mathcal{E} \times \mathcal{M}} \int_{\mathcal{A} \times \mathcal{F}}\left|B_{R}(\epsilon, \mu, \alpha, f)\right|^{2} d P_{e}(\epsilon, \mu) d P_{i}(\alpha, f) .
$$

The problems thus formulated are special cases of classical static zero-sum two player non-cooperative games $[1,12]$ where the evader minimizes over $P_{e} \in \mathcal{P}_{e}$ and the interrogator maximizes over $P_{i} \in \mathcal{P}_{i}$. In such games one 
defines upper and lower values for the game by

$$
\bar{J}=\inf _{P_{e} \in \mathcal{P}_{e}} \sup _{P_{i} \in \mathcal{P}_{i}} J\left(P_{e}, P_{i}\right) \quad \text { and } \quad \underline{J}=\sup _{P_{i} \in \mathcal{P}_{i}} \inf _{P_{e} \in \mathcal{P}_{e}} J\left(P_{e}, P_{i}\right) .
$$

The first represents a security level (worst case scenario) for the evader while the latter is a security level for the interrogator. It is readily argued that $\underline{J} \leq \bar{J}$ and if the equality $J^{*}=\underline{J}=\bar{J}$ holds, then $J^{*}$ is called the value of the game. Moreover, if there exist $P_{e}^{*} \in \mathcal{P}_{e}$ and $P_{i}^{*} \in \mathcal{P}_{i}$ such that

$$
J^{*}=J\left(P_{e}^{*}, P_{i}^{*}\right)=\min _{P_{e} \in \mathcal{P}_{e}} J\left(P_{e}, P_{i}^{*}\right)=\max _{P_{i} \in \mathcal{P}_{i}} J\left(P_{e}^{*}, P_{i}\right),
$$

then $\left(P_{e}^{*}, P_{i}^{*}\right)$ is a saddle point solution or non-cooperative equilibrium of the game.

To investigate theoretical, computational and approximation issues for these problems, it is necessary to put a topology on the space of probability measures. Given a set $I$, a natural choice for $\mathcal{P}(I)$ is the Prohorov metric topology $[14,19$, $21]$ as used for minimization/inverse problems in $[3,4,7,11]$. Prohorov metric ( $\rho$ ) convergence is weak $k^{*}$ convergence in $\mathcal{P}$ when $\mathcal{P}$ is considered as a subset of the topological dual $C_{B}^{*}(I)$ of the space $C_{B}(I)$ of bounded continuous functions on $I$. More precisely, convergence $\rho\left(P_{k}, P\right) \rightarrow 0$ in the Prohorov metric is equivalent to

$$
\int_{I} f(\xi) d P_{k}(\xi) \rightarrow \int_{I} f(\xi) d P(\xi)
$$

for all bounded, uniformly continuous $f: I \rightarrow \mathbb{R}^{1}$.

It is known [3, 4] that if $I$ is a complete metric space, then $\mathcal{P}$ taken with the Prohorov metric is a complete metric space. Moreover, if $I$ is compact, then so is $\mathcal{P}$. Using these properties and arguments similar to those in $[3,6,7,10$, 11], one can develop well-posedness and approximation results for the minmax problems defined above. Efficient computational methods that correspond to von Neumann's finite mixed strategies [1] can readily be presented in this context. These can be based on several approximation theories that have been recently developed and used. The first, developed in [3] and based on Dirac delta measures, is summarized in the following theorem. 
Theorem 2.1. Let I be a complete, separable metric space, $\mathcal{B}$ the class of all Borel subsets of $I$ and $\mathcal{P}(I)$ the space of probability measures on $(I, \mathcal{B})$. Let $I_{0}=\left\{\xi_{j}\right\}_{j=1}^{\infty}$ be a countable, dense subset of $I$. Then the set of $P \in \mathcal{P}(I)$ such that $P$ has finite support in $I_{0}$ and rational masses is dense in $\mathcal{P}(I)$ in the $\rho$ metric. That is,

$$
\begin{gathered}
\mathcal{P}_{0}(I) \equiv\left\{P \in \mathcal{P}(I): P=\sum_{j=1}^{k} p_{j} \Delta_{\xi_{j}}, k \in \mathcal{N}^{+},\right. \\
\left.\xi_{j} \in I_{0}, p_{j} \text { rational, } \sum_{j=1}^{k} p_{j}=1\right\}
\end{gathered}
$$

is dense in $\mathcal{P}(I)$ taken with the $\rho$ metric, where $\Delta_{\xi_{j}}$ is the Dirac measure with atom at $\xi_{j}$.

It is rather easy to use the ideas and results associated with this theorem to develop computationally efficient schemes. Given

$$
I_{d}=\bigcup_{M=1}^{\infty} I_{M} \quad \text { with } \quad I_{M}=\left\{\xi_{j}^{M}\right\}_{j=1}^{M}
$$

(a "partition" of $I$ ) chosen so that $I_{d}$ is dense in $I$, define

$$
\begin{gathered}
\mathcal{P}^{M}(I)=\left\{P \in \mathcal{P}(I): P=\sum_{j=1}^{M} p_{j} \Delta_{\xi_{j}^{M}}, \xi_{j}^{M} \in I_{M},\right. \\
\left.p_{j} \text { rational, } \sum_{j=1}^{M} p_{j}=1\right\} .
\end{gathered}
$$

Then we find

(i) $\mathcal{P}^{M}(I)$ is a compact subset of $\mathcal{P}(I)$ in the $\rho$ metric,

(ii) $\mathcal{P}^{M}(I) \subset \mathcal{P}^{M+1}(I)$ whenever $I_{M+1}$ is a refinement of $I_{M}$,

(iii) " $\mathcal{P}^{M}(I) \rightarrow \mathcal{P}(I)$ " in the $\rho$ topology; that is, for $M$ sufficiently large, elements in $\mathcal{P}(I)$ can be approximated in the $\rho$ metric by elements of $\mathcal{P}^{M}$.

A second class of approximations, based on linear splines, was developed and used in [11] for problems where one assumes that the probability distributions to be approximated possess densities in $L^{2}$. 
Theorem 2.2. Let $\mathcal{F}$ be a weakly compact subset of $L^{2}(I)$, I compact and let $\mathcal{P}_{\mathcal{F}}(I) \equiv\left\{P \in \mathcal{P}(I): P^{\prime}=p, p \in \mathcal{F}\right\}$. Then $\mathcal{P}_{\mathcal{F}}(I)$ is compact in $\mathcal{P}(I)$ in the $\rho$ metric. Moreover, if we define $\left\{\ell_{j}^{M}\right\}$ to be the linear splines on I corresponding to the partition $I_{M}$, where $\bigcup_{M} I_{M}$ is dense I, define

$$
\mathcal{P}^{M} \equiv\left\{p^{M}: p^{M}=\sum_{j} b_{j}^{M} \ell_{j}^{M}, b_{j}^{M} \text { rational }\right\}
$$

and if

$$
\mathcal{P}_{\mathcal{F}^{M}} \equiv\left\{P_{M} \in \mathcal{P}(I): P_{M}=\int p^{M}, p^{M} \in \mathcal{P}^{M}\right\},
$$

we have $\bigcup_{M} \mathcal{P}_{\mathcal{F}^{M}}$ is dense in $\mathcal{P}_{\mathcal{F}}(I)$ taken with the $\rho$ metric.

A study comparing the relative strengths and weaknesses of these two classes of approximation schemes in the context of inverse problems is given in [5].

\section{Theoretical results}

To establish existence of a saddle point solution for the evasion-interrogation problems formulated above, one can employ a fundamental result of von Neumann $[22,23]$ as stated by Aubin ([1]-see p. 126).

Theorem 3.1 (von Neumann). Suppose $X_{0}, Y_{0}$ are compact, convex subsets of metric linear spaces $X, Y$ respectively. Further suppose that

(i) for all $y \in Y_{0}, x \rightarrow f(x, y)$ is convex and lower semi-continuous;

(ii) for all $x \in X_{0}, y \rightarrow f(x, y)$ is concave and upper semi-continuous.

Then there exists a saddle point $\left(x^{*}, y^{*}\right)$ such that

$$
f\left(x^{*}, y^{*}\right)=\min _{X_{0}} \max _{Y_{0}} f(x, y)=\max _{Y_{0}} \min _{X_{0}} f(x, y) .
$$

A straight forward application of these results to the problems under discussion lead immediately to desired well-posedness results. 
Theorem 3.2. Suppose $\mathcal{E}, \mathcal{M}, \Phi, \mathcal{F}$ are compact and the spaces $X_{0}=\mathcal{P}(\mathcal{E} \times$ $\mathcal{M}), Y_{0}=\mathcal{P}(\Phi \times \mathcal{F})$ are taken with the Prohorov metric. Then $X_{0}, Y_{0}$ are compact, convex subsets of $X=C_{B}^{*}(\mathcal{E} \times \mathcal{M})$ and $Y=C_{B}^{*}(\Phi \times \mathcal{F})$, respectively. Moreover, there exists $\left(P_{e}^{*}, P_{i}^{*}\right) \in \mathcal{P}(\mathcal{E} \times \mathcal{M}) \times \mathcal{P}(\Phi \times \mathcal{F})$ such that

$$
J\left(P_{e}^{*}, P_{i}^{*}\right)=\min _{\mathcal{P}(\mathcal{E} \times \mathcal{M})} \max _{\mathcal{P}(\Phi \times \mathcal{F})} J\left(P_{e}, P_{i}\right)=\max _{\mathcal{P}(\Phi \times \mathcal{F})} \min _{\mathcal{P}(\mathcal{E} \times \mathcal{M})} J\left(P_{e}, P_{i}\right) .
$$

For computations, we may use the "delta" approximations of Theorem 2.1 and obtain

$$
\begin{aligned}
d P_{e}(\epsilon, \mu) & \approx \sum p_{e}^{j} \delta_{\left(\epsilon_{j}, \mu_{j}\right)}(\epsilon, \mu) d \epsilon d \mu \\
d P_{i}(\phi, f) & \approx \sum p_{i}^{j} \delta_{\left(\phi_{j}, f_{j}\right)}(\phi, f) d \phi d f
\end{aligned}
$$

As noted above, a convergence theory can be found in [3]. This corresponds precisely to von Neumann's finite mixed strategies framework for protection by disguising intentions from opponents, i.e., by introducing uncertainty in the players' choices.

To illustrate the computational framework based on the delta measure approximations, we take

$$
\begin{aligned}
& d P_{e}^{M}(\epsilon, \mu)=\sum_{j=1}^{M} p_{j}^{M} \delta_{\left(\epsilon_{j}^{M}, \mu_{j}^{M}\right)} d \epsilon d \mu \quad \text { and } \\
& d P_{i}^{N}(\phi, f)=\sum_{k=1}^{N} q_{k}^{N} \delta_{\left(\phi_{k}^{N}, f_{k}^{N}\right)} d \phi d f
\end{aligned}
$$

which can be represented respectively by

$$
\bar{p}^{M}=\left\{p_{j}^{M}\right\}_{j=1}^{M} \in P^{M} \equiv\left\{\bar{p} \in \mathbb{R}^{M} \mid p_{j} \geq 0, \sum_{j=1}^{M} p_{j}=1\right\}
$$

and

$$
\bar{q}^{N}=\left\{q_{k}^{N}\right\}_{k=1}^{N} \in Q^{N} \equiv\left\{\bar{q} \in \mathbb{R}^{N} \mid q_{k} \geq 0, \sum_{k=1}^{N} q_{k}=1\right\} .
$$


We note that in this case the $p_{j}^{M}, q_{k}^{N}$ are the probabilities associated with the use of the material parameters $\left(\epsilon_{j}^{M}, \mu_{j}^{M}\right)$ and interrogating parameters $\left(\phi_{k}^{N}, f_{k}^{N}\right)$ in the mixed strategies of the evader and interrogator, respectively.

Then $J\left(P_{e}^{M}, P_{i}^{N}\right)$ reduces to

$$
\mathcal{J}\left(\bar{p}^{M}, \bar{q}^{N}\right)=\sum_{k=1}^{N} \sum_{j=1}^{M} p_{j}^{M}\left|B_{R}\left(\epsilon_{j}^{M}, \mu_{j}^{M}, \phi_{k}^{N}, f_{k}^{N}\right)\right|^{2} q_{k}^{N}
$$

where $B_{R}$ is a measure of the reflected field (either the reflection coefficient or the far field scattering intensity). Since $P^{M}, Q^{N}$ are compact, convex subsets of $\mathbb{R}^{M}, \mathbb{R}^{N}$, respectively, we have the following theorem.

Theorem 3.3. For fixed $M, N$ there exists $\left(\bar{p}_{*}^{M}, \bar{q}_{*}^{N}\right)$ in $P^{M} \times Q^{N}$ such that

$$
\begin{aligned}
\mathcal{J}^{*}=\mathcal{J}\left(\bar{p}_{*}^{M}, \bar{q}_{*}^{N}\right) & =\min _{\bar{p}^{M} \in P^{M}} \max _{\bar{q}^{N} \in Q^{N}} \mathcal{J}\left(\bar{p}^{M}, \bar{q}^{N}\right) \\
& =\max _{\bar{q}^{N} \in Q^{N}} \min _{\bar{p}^{M} \in P^{M}} \mathcal{J}\left(\bar{p}^{M}, \bar{q}^{N}\right) .
\end{aligned}
$$

Assume further that $(\epsilon, \mu, \phi, f) \rightarrow B_{R}(\epsilon, \mu, \phi, f)$ is continuous on $\mathcal{E} \times$ $\mathcal{M} \times \Phi \times \mathcal{F}$ which is assumed compact. Then there exists a sequence $\left(\bar{p}_{*}^{M}, \bar{q}_{*}^{N}\right)$ of elements from $P^{M} \times Q^{N}$ respectively with corresponding $\left(P_{e}^{M}, P_{i}^{N}\right)$ in $\mathcal{P}(\mathcal{E} \times \mathcal{M}) \times \mathcal{P}(\Phi \times \mathcal{F})$ converging in the Prohorov metric to $\left(P_{e}^{*}, P_{i}^{*}\right)$ which is a saddle point for the original minmax problem.

Proof. The hypothesis that the map $(\epsilon, \mu, \phi, f) \rightarrow B_{R}(\epsilon, \mu, \phi, f)$ be continuous on $\mathcal{E} \times \mathcal{M} \times \Phi \times \mathcal{F}$ implies continuity of the map $\left(P_{e}, P_{i}\right) \rightarrow J\left(P_{e}, P_{i}\right)$ on $X_{0} \times Y_{0} \rightarrow \mathbb{R}_{+}^{1}$, where $X_{0}=\mathcal{P}(\mathcal{E} \times \mathcal{M})$ and $Y_{0}=\mathcal{P}(\Phi \times \mathcal{F})$ are compact convex in $X=C_{B}^{*}(\mathcal{E} \times \mathcal{M})$ and $Y=C_{B}^{*}(\Phi \times \mathcal{F})$, respectively. This is due to the compactness of $\mathcal{E} \times \mathcal{M}$ and $\Phi \times \mathcal{F}$, and properties of the Prohorov metric.

Let $P^{M}, Q^{N}$ be defined as in (6), (7), and observe that these are compact convex subsets of $\mathbb{R}^{M}, \mathbb{R}^{N}$, respectively. Now let $P_{e} \in X_{0}$ and $P_{i} \in Y_{0}$ be arbitrary. Then by Theorem 2.1 and (iii) above (see also [3]), there exists a sequence $\bar{p}^{M}, \bar{q}^{N} \in P^{M}, Q^{N}$ with associated measures $P_{e}^{M}, P_{i}^{N}$, respectively, such that

$$
P_{e}^{M} \rightarrow P_{e} \text { in } X_{0} \text { as } M \rightarrow \infty \text { and } P_{i}^{N} \rightarrow P_{i} \text { in } Y_{0} \text { as } N \rightarrow \infty .
$$


Let $P_{e}^{M *}, P_{i}^{N *}$ (guaranteed to exist by continuity, compactness, and the von Neumann theorem) with coordinates $\bar{p}_{*}^{M}, \bar{q}_{*}^{N}$, respectively, satisfy

$$
J\left(P_{e}^{M *}, P_{i}^{N}\right) \leq J\left(P_{e}^{M *}, P_{i}^{N *}\right) \leq J\left(P_{e}^{M}, P_{i}^{N *}\right)
$$

for all $\left(P_{e}^{M}, P_{i}^{N}\right)$ in $X_{0} \times Y_{0}$ with coordinate representations in $P^{M}, Q^{N}$, respectively. Now $X_{0} \times Y_{0}$ compact implies that there exists a subsequence $\left(P_{e}^{M_{k^{*}}}, P_{i}^{N_{k} *}\right)$ and $\left(\tilde{P}_{e}, \tilde{P}_{i}\right)$ in $X_{0} \times Y_{0}$ such that $\left(P_{e}^{M_{k^{*}}}, P_{i}^{N_{k} *}\right) \rightarrow\left(\tilde{P}_{e}, \tilde{P}_{i}\right)$ in $X_{0} \times Y_{0}$.

Consider (8) for indices $M_{k}, N_{k}$, so that

$$
J\left(P_{e}^{M_{k^{*}}}, P_{i}^{N_{k}}\right) \leq J\left(P_{e}^{M_{k^{*}}}, P_{i}^{N_{k} *}\right) \leq J\left(P_{e}^{M_{k}}, P_{i}^{N_{k} *}\right) .
$$

Then given the continuity of $J$ and taking the limit we find

$$
J\left(\tilde{P}_{e}, P_{i}\right) \leq J\left(\tilde{P}_{e}, \tilde{P}_{i}\right) \leq J\left(P_{e}, \tilde{P}_{i}\right) .
$$

Since $P_{e}, P_{i}$ are arbitrary in $X_{0}, Y_{0}$ respectively, we have $\left(\tilde{P}_{e}, \tilde{P}_{i}\right)$ is a saddle point for the original minmax problem.

Further note that since these arguments hold for any subsequence of $\left(P_{e}^{M *}\right.$, $\left.P_{i}^{N *}\right)$, then

$$
J\left(P_{e}^{M *}, P_{i}^{N}\right) \leq J\left(P_{e}^{M *}, P_{i}^{N *}\right) \leq J\left(P_{e}^{M}, P_{i}^{N *}\right)
$$

and $P_{e}^{M *} \rightarrow \tilde{P}_{e}$ as well as $P_{i}^{N *} \rightarrow \tilde{P}_{i}$. That is, any subsequence of $P_{e}^{M *}, P_{i}^{N *}$ has a convergent subsequence to $\tilde{P}_{e}, \tilde{P}_{i}$ so that the sequence itself must converge to $\tilde{P}_{e}, \tilde{P}_{i}$.

\section{Preliminary numerical calculations}

In [9], the authors considered a number of different material designs in their investigation of counter interrogation and counter-counter interrogation feasibility. We use a number of these materials to define families of admissible evader sets $\mathcal{E} \times \mathcal{M}$ in our initial exploratory calculations in demonstrating ease of calculations for problems as outlined above in Theorem 3.3 for the delta measures as defined in (6) and (7). The materials we use here were designed in [9] in the 
following way. For a given assumed interrogator design frequency set $I_{D}$ and assumed normal incident waves (incident angle $\phi=0$ ), the evader designs a material by optimizing over admissible sets for $\epsilon$ and $\mu$, the criteria

$$
J_{\infty}(\epsilon, \mu)=\max _{f \in I_{D}} B_{R}(\epsilon, \mu, 0, f)
$$

to obtain "optimal" coating parameters $\left(\epsilon^{*}, \mu^{*}\right)$. With the thickness $d=2.5$ millimeters, Fresnel's formula (1)-(2) was used to obtain a series of materials labelled $1 R, 2 R, \ldots, 6 R$ corresponding to $\left(\epsilon^{*}, \mu^{*}\right)=\left(\epsilon_{j R}, \mu_{j R}\right)$ in [9]. Adopting the same labelling here, we allow the evader to choose from subsets composed of elements from the following six materials denoted by their respective parameter values $\left(\epsilon_{j R}, \mu_{j R}\right), j=1, \ldots, 6$ :

1R: $\left(\epsilon_{1 R}, \mu_{1 R}\right)=(900.4+38.2 i, 1)$ optimizing only over $\epsilon$ with $\mu=1$ fixed and $I_{D}=\{1 G H z\}$,

$2 R:\left(\epsilon_{2 R}, \mu_{2 R}\right)=(90.7+21.4 i, 10+2 i)$ for $I_{D}=\{1 G H z\}$,

$3 R:\left(\epsilon_{3 R}, \mu_{3 R}\right)=(90.7+21.4 i, 10+2 i)$ for $I_{D}=[0.8,1.25]$,

$4 R:\left(\epsilon_{4 R}, \mu_{4 R}\right)=(5625.4+95.5 i, 1)$ optimizing only over $\epsilon$ with $\mu=1$ fixed and $I_{D}=\{0.4 G H z\}$,

$5 R:\left(\epsilon_{5 R}, \mu_{5 R}\right)=(558.6+29.3 i, 10+1.2 i)$ for $I_{D}=\{0.4 \mathrm{GHz}\}$,

6R: $\left(\epsilon_{6 R}, \mu_{6 R}\right)=(549.3+150 i, 10+2 i)$ for $I_{D}=[0.32,0.5]$.

Graphs of the resulting reflection coefficients $B_{R}\left(\epsilon_{j R}, \mu_{j R}, 0, f\right)=R\left(\epsilon_{j R}\right.$, $\left.\mu_{j R}, f\right)$ are plotted as a function of possible interrogating frequency in Figure 2 . Note that in the cases $1 R, 2 R, 4 R, 5 R$, if the interrogator uses the design frequency, the evader has a high likelihood of avoiding detection, but his/her evasion strategy is easily defeated by an interrogating signal away from the design frequency.

\subsection{Typical results}

We carried out a number of different optimization trials using these materials with the "delta" measure approximations outlined above. We present in Table 1 

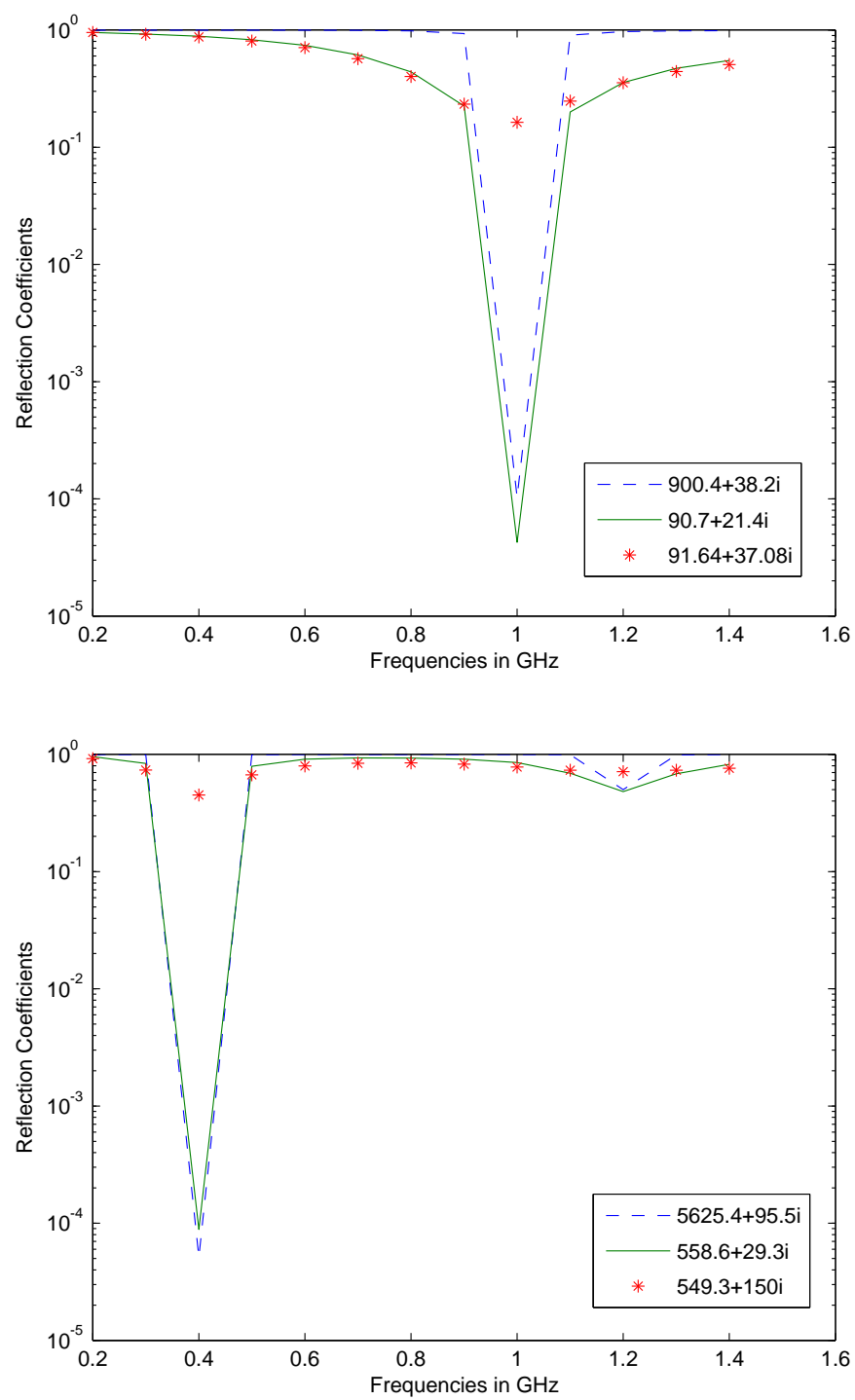

Figure 2 - Reflection coefficients calculated using $\left(\epsilon_{j R}, \mu_{j R}\right)$ from materials $1 \mathrm{R}$, $2 \mathrm{R}$, and $3 \mathrm{R}$ which are associated with $(900.4+38.2 \mathrm{i}, 1),(90.7+21.4 \mathrm{i}, 10+2 \mathrm{i})$, and $(91.64+37.08 \mathrm{i}, 10+2 \mathrm{i})$, respectively (top) and materials $4 \mathrm{R}, 5 \mathrm{R}$, and $6 \mathrm{R}$ which are associated with $(5625.4+95.5 \mathrm{i}, 1),(558.6+29.3 \mathrm{i}, 10+1.2 \mathrm{i})$, and $(549.3+150 \mathrm{i}, 10+2 \mathrm{i})$, respectively (bottom). 
a sample of results obtained for several choices of admissible evader material sets $\mathcal{E} \times \mathcal{M}$ and interrogator frequency sets $\mathcal{F}$. The minmax calculations are readily carried out using standard MATLAB minimization packages.

\begin{tabular}{|l|l|}
\hline \multicolumn{1}{|c|}{ Evader Set } & \multicolumn{1}{c|}{ Interrogator Set } \\
\hline $\mathcal{E} \times \mathcal{M}=\left\{\left(\epsilon_{j R}, \mu_{j R}\right): j=1,2,3\right\}$ & $\mathcal{F}=\{.4, .6,1\}$ \\
\hline $\mathcal{E} \times \mathcal{M}=\left\{\left(\epsilon_{j R}, \mu_{j R}\right): j=1,2,3\right\}$ & $\mathcal{F}=\{.4,1\}$ \\
\hline $\mathcal{E} \times \mathcal{M}=\left\{\left(\epsilon_{j R}, \mu_{j R}\right): j=4,5,6\right\}$ & $\mathcal{F}=\{.4, .6\}$ \\
\hline $\mathcal{E} \times \mathcal{M}=\left\{\left(\epsilon_{j R}, \mu_{j R}\right): j=4,5,6\right\}$ & $\mathcal{F}=\{.4, .6,1\}$ \\
\hline \hline \multicolumn{2}{|c|}{ Results } \\
\hline $\bar{p}_{*}^{M}=(0,0,1)$ & $\bar{q}_{*}^{N}=(1,0,0)$ \\
\hline $\bar{p}_{*}^{M}=(0,0.241,0.759)$ & $\bar{q}_{*}^{N}=(1,0)$ \\
\hline $\bar{p}_{*}^{M}=(0,0.667,0.333)$ & $\bar{q}_{*}^{N}=(0,1)$ \\
\hline $\bar{p}_{*}^{M}=(0,0.667,0.333)$ & $\bar{q}_{*}^{N}=(0,0.667,0.333)$ \\
\hline
\end{tabular}

Table 1 - Results of the saddle point calculations for a sample of evader sets $\mathcal{E} \times \mathcal{M}$ and interrogator sets $\mathcal{F}$.

A few words on the findings in the table are in order. First we note that when materials $1 R, 2 R, 3 R$ (all of which are based on an $I_{D}$ of $1 \mathrm{GHz}$ or an interval around $1 \mathrm{GHz}$ ) are available to the evader, the best strategy for the interrogator is to always choose (with probability 1) the interrogating frequency $(.4 \mathrm{G} \mathrm{Hz}$ ) furthermost from the design frequencies. The evader's choice is slightly more delicate. In the first row of Table 1 the evader always chooses material $3 R$ which is based on design for the largest $I_{D}$ among $1 R, 2 R, 3 R$. However, in the second row we see that the evader should use $2 R$ for $24.1 \%$ of the time and $3 R$ the other $75.9 \%$ of the time. If the materials $4 R, 5 R, 6 R$ are available, the evader should use material $5 R$ for $66.7 \%$ of the time and material $6 R$ the remainder of the time. In this situation the interrogator should use $f=.6 \mathrm{GHz}$ if only the frequencies $\{.4, .6\}$ are available. If $\{.4, .6,1\}$ are available, the interrogator should use $f=.6 \mathrm{GHz}$ for $66.7 \%$ of his interrogations and $f=1 \mathrm{GHz}$ for $33.3 \%$ of the interrogations to maximize his chances of detecting the evader. 


\section{Concluding remarks}

In this note we have presented preliminary ideas for evader-interrogator strategies in electromagnetic counter and counter-counter interrogation games. While the ideas are simple, they possess potential for readily implementable strategies in a number of other modern non-cooperative adversarial situations in that similar problems arise in information-misinformation dissemination, data retrieval and analysis, and internet traffic and security. The computational results we present are extremely simple, but illustrate aptly the potential. More realistic problems (currently under investigation) involve multiple layers of materials and the corresponding Fresnel's formulae (see [13, 16, 24]) for multiple stacked layers of dielectric materials. And of course one should investigate larger values of $M$ and $N$ than the values $M=3$ and $N=2$ or 3 used in the preliminary calculations reported on above.

In our computational examples, $P_{e}$ and $P_{i}$ were taken as discrete measures with a finite number of atoms, i.e., finite weighted combinations of the delta measures. Our current computational efforts are on absolutely continuous measures $d P_{e}(\epsilon, \mu)=p(\epsilon, \mu) d \epsilon d \mu$ and $d P_{i}(f)=q(f) d f$ using the linear spline approximations presented in Theorem 2.2. Specifically, these can be used to obtain approximations

$$
\begin{gathered}
p(\epsilon, \mu) \approx p^{M}(\epsilon, \mu)=\sum_{j=1}^{M} p_{j}^{M} \ell_{j}^{M}(\epsilon) \ell_{j}^{M}(\mu), \\
q(f) \approx q^{N}(f)=\sum_{k=1}^{N} q_{k}^{N} \ell_{k}^{N}(f)
\end{gathered}
$$

which again reduces considerations to a simple minmax problem for

$$
\mathcal{J}\left(\bar{p}^{M}, \bar{q}^{N}\right)=\sum_{k=1}^{N} \sum_{j=1}^{M} p_{j}^{M} A_{j k}^{M N} q_{k}^{N},
$$

where now

$$
A_{j k}^{M N}=\iint_{\mathcal{E} \times \mathcal{M} \times \mathcal{F}} \int \ell_{j}^{M}(\epsilon) \ell_{j}^{M}(\mu)|R(\epsilon, \mu, f)|^{2} \ell_{k}^{N}(f) d \epsilon d \mu d f .
$$


More generally, one should consider approximations

$$
p(\epsilon, \mu) \approx p^{I M}(\epsilon, \mu)=\sum_{i=1}^{I} \sum_{j=1}^{M} p_{i j}^{I M} \ell_{i}^{I}(\epsilon) \ell_{j}^{M}(\mu),
$$

resulting in a tensor product in the approximating minmax problems with tensor

$$
A_{i j k}^{I M N}=\iint_{\mathcal{E} \times \mathcal{M} \times \mathcal{F}} \int \ell_{i}^{I}(\epsilon) \ell_{j}^{M}(\mu)|R(\epsilon, \mu, f)|^{2} \ell_{k}^{N}(f) d \epsilon d \mu d f .
$$

Of course, in these approximations the $p^{\prime} s$ and $q^{\prime} s$ are no longer simple probabilities (as in the case of the delta basis elements), but are weighting coefficients for the density basis elements $\left\{\ell_{j}^{M}\right\}$, etc., and must be properly constrained so that the finite combinations sum to a probability density.

Acknowledgement. This research was supported in part by the U.S. Air Force Office of Scientific Research under grant AFOSR FA9550-04-1-0220. The authors would like to thank Dr. Richard Albanese of the AFRL, Brooks AFB, San Antonio, TX, for numerous stimulating and valuable comments and suggestions during the course of this effort.

\section{REFERENCES}

[1] J.-P. Aubin, Optima and Equilbria: An Introduction to Nonlinear Analysis, Spring-Verlag, Berlin Heidelberg, 1993.

[2] A. Bamberger, P. Joly and J.E. Roberts, Second-order absorbing boundary conditions for the wave equation: a solution for the corner problem. SIAM J. Numer. Anal., 27 (1990), 323-352.

[3] H.T. Banks and K.L. Bihari, Modeling and estimating uncertainty in parameter estimation. Inverse Problems, 17 (2001), 95-111.

[4] H.T. Banks, D. Bortz, G.A. Pinter and L.K. Potter, Modeling and imaging techniques with potential for application in bioterrorism, Chapter 6 in Bioterrorism: Mathematical Modeling Applications in Homeland Security, H.T. Banks and C. Castillo-Chavez, eds., Frontiers in Applied Mathematics, SIAM, Philadelphia, 2003, pp. 129-154.

[5] H.T. Banks and J.L. Davis, A comparison of approximation methods for the estimation of probability distributions on parameters, Tech. Report CRSC-TR05-38, Center for Research in Scientific Computation, N.C. State University, Raleigh, NC, October, 2005; Applied Numerical Mathematics, to appear. 
[6] H.T. Banks and N.L. Gibson, Well-posedness of solutions with a distribution of dielectric parameters. Applied Mathematics Letters, 18 (2005), 423-430.

[7] H.T. Banks and N.L. Gibson, Electromagnetic inverse problems involving distributions of dielectric mechanisms and parameters, Tech. Report CRSC-TR05-29, Center for Research in Scientific Computation, N.C. State University, Raleigh, NC, August, 2005. Quarterly Applied Math., to appear.

[8] H.T. Banks, K. Ito, G.M. Kepler and J.A. Toivanen, Material surface design to counter electromagnetic interrogation of targets, Tech. Report CRSC-TR04-37, Center for Research in Scientific Computation, N.C. State University, Raleigh, NC, November, 2004. SIAM J. Appl. Math., 66 (2006), 1027-1049.

[9] H.T. Banks, K. Ito and J.A. Toivanen, Determination of interrogating frequencies to maximize electromagnetic backscatter from objects with material coatings, Tech. Report CRSCTR05-30, Center for Research in Scientific Computation, N.C. State University, Raleigh, NC, August, 2005. Communications in Comp. Physics, 1 (2006), 357-377.

[10] H.T. Banks and K. Kunisch, Estimation Techniques for Distributed Parameter Systems, Birkhauser, Boston, 1989.

[11] H.T. Banks and G.A. Pinter, A probabilistic multiscale approach to hysteresis in shear wave propagation in biotissue. SIAM J. Multiscale Modeling and Simulation, 3 (2005), 395-412.

[12] T. Basar and G.J. Olsder, Dynamic Noncooperative Game Theory, SIAM Publ., Philadelphia, PA, $2^{\text {nd }}$ edition, 1999.

[13] S. Beeler, H.T. Tran and N. Dietz, Representation of GaP formation by a reduced order surface kinetics model using p-polarized reflectance measurements. J. Appl. Physics, 86 (1999), 674-682.

[14] P. Billingsley, Convergence of Probability Measures, John Wiley, New York, 1968.

[15] D. Colton and R. Kress, Inverse Acoustic and Electromagnetic Scattering Theory, Vol. 93 of Applied Mathematical Sciences, Springer-Verlag, Berlin, $2^{\text {nd }}$ ed., 1998.

[16] O.S. Heavens, Optical Properties of Thin Solid Films, Butterworths, London, 1955.

[17] E. Heikkola, Y.A. Kuznetsov, P. Neittaanmäki and J. Toivanen, Fictitious domain methods for the numerical solution of two-dimensional scattering problems. J. Comput. Phys., 145 (1998), 89-109.

[18] E. Heikkola, T. Rossi and J. Toivanen, Fast direct solution of the Helmholtz equation with a perfectly matched layer or an absorbing boundary condition. Internat. J. Numer. Methods Engr., 57 (2003), 2007-2025.

[19] P.J. Huber, Robust Statistics, Wiley \& Sons, New York, 1981.

[20] J.D. Jackson, Classical Electrodynamics, Wiley \& Sons, New York, 1975. 
[21] Yu. V. Prohorov, Convergence of random processes and limit theorems in probability theory, Theor. Prob. Appl., 1 (1956), 157-214.

[22] J. von Neumann, Zur theorie der gesellschaftsspiele. Math. Ann., 100 (1928), 295-320.

[23] J. von Neumann and O. Morgenstern, Theory of Games and Economic Behavior, Princeton University Press, Princeton, NJ, 1944.

[24] L. Ward, The Optical Constants of Bulk Materials and Films, IOP Press, London, 1994. 